Western University

Scholarship@Western

$12-7-2008$

\title{
Scan-rescan and intra-observer variability of magnetic resonance imaging of carotid atherosclerosis at 1.5 T and 3.0 T
}

Arvin Vidal

Yves Bureau

Trevor Wade

J David Spence

Western University, dspence@robarts.ca

Brian K Rutt

See next page for additional authors

Follow this and additional works at: https://ir.lib.uwo.ca/biophysicspub

Part of the Medical Biophysics Commons

Citation of this paper:

Vidal, Arvin; Bureau, Yves; Wade, Trevor; Spence, J David; Rutt, Brian K; Fenster, Aaron; and Parraga, Grace, "Scan-rescan and intra-observer variability of magnetic resonance imaging of carotid atherosclerosis at 1.5 T and 3.0 T" (2008). Medical Biophysics Publications. 132.

https://ir.lib.uwo.ca/biophysicspub/132 


\section{Authors}

Arvin Vidal, Yves Bureau, Trevor Wade, J David Spence, Brian K Rutt, Aaron Fenster, and Grace Parraga

This article is available at Scholarship@Western: https://ir.lib.uwo.ca/biophysicspub/132 
Scan-rescan and intra-observer variability of magnetic resonance imaging of carotid atherosclerosis at $1.5 \mathrm{~T}$ and $3.0 \mathrm{~T}$

To cite this article: Arvin Vidal et al 2008 Phys. Med. Biol. 536821

View the article online for updates and enhancements.

\section{Related content}

$$
\begin{aligned}
& \text { - } \frac{\text { Carotid wall volume quantification from }}{\text { magnetic resonance images using }} \\
& \frac{\text { deformable model fitting and learning- }}{\text { based correction of systematic errors }} \\
& \hline \text { K Hameeteman, R van 't Klooster, M } \\
& \text { Selwaness et al. } \\
& \text { - Variability in ultrasound volume } \\
& \frac{\text { measurement of murine liver metastases }}{\text { L A Wirtzfeld, K C Graham, A C Groom et }} \\
& \text { al. } \\
& \text { - Novel 3D ultrasound image-based } \\
& \frac{\text { biomarkers based on a feature selection }}{\text { from a 2D standardized vessel wall }} \\
& \frac{\text { thickness map: a tool for sensitive }}{\text { assessment of therapies for carotid }} \\
& \frac{\text { atherosclerosis }}{\text { Bernard Chiu, Bing Li and Tommy W S }} \\
& \text { Chow }
\end{aligned}
$$

\section{Recent citations}

\section{- Jie Sun and Thomas S. Hatsukami}

Comparison of two different measurement methods in evaluating basilar atherosclerotic plaque using highresolution MRI at 3 tesla Luguang Chen et al

Effects of Pitavastatin on Lipid-rich Carotid Plaques Studied Using High-resolution Magnetic Resonance Imaging Tao Feng et al

\section{RayStation}

\section{RAYSTATION NOW WITH MACHINE LEARNING}

SEE MORE 》 


\title{
Scan-rescan and intra-observer variability of magnetic resonance imaging of carotid atherosclerosis at $1.5 \mathrm{~T}$ and $3.0 \mathrm{~T}$
}

\author{
Arvin Vidal $^{1,2}$, Yves Bureau ${ }^{3}$, Trevor Wade ${ }^{1,3}$, J David Spence $^{4}$, \\ Brian K Rutt ${ }^{1,2,3}$, Aaron Fenster ${ }^{1,2,3}$ and Grace Parraga ${ }^{1,2,3,5}$ \\ ${ }^{1}$ Imaging Research Laboratories, Robarts Research Institute, 100 Perth Drive, London, \\ N6A 5K8 Canada \\ 2 Department of Medical Biophysics, The University of Western Ontario, London, N6A 5C1 \\ Canada \\ ${ }^{3}$ Graduate Program in Biomedical Engineering, The University of Western Ontario, London, \\ N6A 5B9 Canada \\ ${ }^{4}$ Stroke Prevention and Atherosclerosis Research Centre, Robarts Research Institute, \\ 1400 Western Road, London, N6A 5K8 Canada \\ E-mail: avidal@imaging.robarts.ca,ybureau@lawsonimaging.ca,twade@imaging.robarts.ca, \\ DSpence@robarts.ca, brutt@imaging.robarts.ca, afenster@imaging.robarts.ca and \\ gep@imaging.robarts.ca
}

Received 26 April 2008, in final form 14 August 2008

Published 12 November 2008

Online at stacks.iop.org/PMB/53/6821

\begin{abstract}
Carotid atherosclerosis measurements for eight subjects at baseline and $14 \pm$ 2 days later were examined using $1.5 \mathrm{~T}$ and 3.0 T magnetic resonance imaging (MRI). A single observer blinded to field strength, subject and timepoint manually segmented carotid artery wall and lumen boundaries in randomized images in five measurement trials. Mean increases in the signal-to-noise ratios (SNR) for T1-weighted images acquired at 3.0 T compared to $1.5 \mathrm{~T}$ were $90 \%$ (scan) and $80 \%$ (rescan). Despite significantly improved SNR and contrast-tonoise ratios (CNR) for images acquired at 3.0 T, vessel wall volume (VWV) intra-observer variability was not significantly different using coefficients of variation (COV), and intraclass correlation coefficients (ICC). VWV interscan variability and consistency at both field strengths were not statistically different $\left(1.5 \mathrm{~T} / 3.0 \mathrm{~T} \mathrm{COV}=5.7 \% / 7.8 \%, R^{2}=0.96\right.$ for $1.5 \mathrm{~T}$ and $R^{2}=0.87$ for 3.0 T). A two-way analysis of variance showed a VWV dependence on field strength but not scan timepoint. In addition, a paired $t$-test showed significant differences in VWV measured at 3.0 T as compared to $1.5 \mathrm{~T}$. These results suggest that although images acquired at 1.5 T have lower SNR and CNR VWV, measurement variability was not significantly different from 3.0 T VWV and
\end{abstract}

5 Author to whom any correspondence should be addressed. 
that VWV is field-strength dependent which may be an important consideration for longitudinal studies.

(Some figures in this article are in colour only in the electronic version)

\section{Introduction}

Atherosclerosis is a progressive disease starting early in life and remaining clinically silent for many years. Many of the landmark studies of clinical atherosclerosis progression have relied on serial monitoring of coronary and carotid luminal stenosis using angiography and these have provided clarity regarding risk (reviewed in Blankenhorn et al (1987), Blankenhorn and Hodis (1994), Brensike et al (1984) and Arntzenius et al (1985)), as well as clear guidelines for the stratification of patients to treatment (Barnett et al 1998). Currently, longitudinal studies of carotid atherosclerosis progression can be undertaken using a variety of other non-invasive imaging techniques such as B-mode ultrasound (measuring intimamedia thickness, reviewed in Barth (2004) and Ashrafian et al (2007)), three-dimensional ultrasound (3DUS) measuring the vessel wall volume (VWV) (Egger et al 2007), the total plaque volume (TPV) (Hatsukami et al 1994, Landry et al 2004, 2005, Steinke and Hennerici 1989) and magnetic resonance imaging (MRI), which allows for the measurement of plaque and arterial wall components (reviewed in Yuan et al (2006)). Longitudinal studies using MRI are dependent upon both the high spatial resolution afforded by the technique as well as high scan-rescan reproducibility. Currently, most MR studies of carotid disease have been undertaken at field strengths of $1.5 \mathrm{~T}$ and it is expected that higher field strengths would provide both improved intra-observer and interscan reproducibility based on the expected improvement in the signal-to-noise ratio (SNR) and contrast-to-noise ratio (CNR) (Cury et al 2006, Koktzoglou et al 2006, Yarnykh et al 2006). A number of recent studies have shown that the SNR and contrast-to-noise (CNR) ratios were higher in carotid images acquired at $3.0 \mathrm{~T}$ as compared to $1.5 \mathrm{~T}$ (Cury et al 2006, Koktzoglou et al 2006, Yarnykh et al 2006). Another recent analysis of carotid atherosclerosis has also specifically assessed reproducibility at 3.0 T (Dehnavi et al 2007) and showed an interscan-intraclass correlation coefficient (ICC) of 0.99 and inter-observer ICC of 0.98 for the measurement of total vessel wall area. For longitudinal studies designed to monitor disease changes during specific interventions and as well in cohort studies, it is necessary to assess interscan (scan-rescan) reliability in order to gain an understanding of the variability that arises during repeated scanning visits. In the prospective study described here, carotid MRI assessments at $1.5 \mathrm{~T}$ and $3.0 \mathrm{~T}$ were examined in subjects with moderate atherosclerosis scanned twice in 2 weeks in order to assess intraobserver and interscan variability within a scan-rescan paradigm simulating typical variability observed in longitudinal studies. MRI-derived VWV was measured on five separate occasions $(8$ subjects $\times 2$ sides $\times 5$ measurements $\times 2$ timepoints $\times 2$ field strengths $=320$ measurements) by an observer who was blinded to subject identity, field strength and timepoint. Intra-observer and interscan reproducibility as well as absolute volumetric measurements derived from T1-weighted black blood MR images acquired at 3.0 T and 1.5 T were directly compared in these study subjects. 
Table 1. Magnetic resonance imaging parameters for T1-weighted double inversion recovery scans at $1.5 \mathrm{~T}$ and $3.0 \mathrm{~T}$.

\begin{tabular}{lll}
\hline $\begin{array}{l}\text { Acquisition parameters for axial } \\
\text { T1 double inversion recovery }\end{array}$ & $1.5 \mathrm{~T}$ & $3.0 \mathrm{~T}$ \\
\hline TE $(\mathrm{ms})$ & 12 & 11.4 \\
TR & $1 \mathrm{RR}$ & $1 \mathrm{RR}$ \\
RBW (kHz) & 41.67 & 41.67 \\
FOV $(\mathrm{cm})$ & 11 & 11 \\
Thickness (mm) & 2 & 2 \\
Matrix & $224 \times 224$ & $224 \times 224$ \\
NEX & 3 & 3 \\
Scan time (minutes) & $8: 48$ & $8: 48$ \\
Fat saturation & Yes & Yes \\
Spacing overlap & 0 & 0 \\
Number of slices & 16 & 16 \\
Pulse sequence & FSE & FSE \\
\hline
\end{tabular}

TE is the echo time, TR is the recovery time, RBW is the receiver bandwidth, FOV is the field of view, NEX is the number of excitations.

\section{Methods}

\subsection{Study subjects}

Ten subject volunteers with carotid total plaque area $\geqslant 0.5 \mathrm{~cm}^{2}$ (previously measured using B-mode ultrasound) were recruited from the Premature Atherosclerosis Clinic and the Stroke Prevention Clinic at University Hospital, London Health Sciences Centre, London, Canada, as previously described (Egger et al 2007). All subjects provided written informed consent to the study protocol approved by the University of Western Ontario Standing Board of Human Research Ethics.

\subsection{Imaging}

All subjects were scanned at 1.5 T and 3.0 T on GE EXCITE whole-body MR systems, with pulse sequences optimized for the associated field strengths but matching in all other aspects and matching custom-built 6-element carotid-bifurcation-optimized receive-only phased-array coils. Both receive-only coils were geometrically and electronically identical, but with one tuned for use at $1.5 \mathrm{~T}$ and the other for use at 3.0 T. For both scanners, a specialized foam head holder was also used to reduce positioning variability and to provide comfortable but secure immobilization of each subject's head. The time frame between MRI scanning sessions at each field strength was approximately $2 \mathrm{~h}$ and all subjects were scanned at baseline (scan) and $14 \pm 2$ days later (rescan) at both field strengths. A 2 week gap between scanning sessions was chosen to minimize subject inconvenience, and to model typical sources of variability stemming from the acquisition of the images due to MR coil positioning, subject motion and subject repositioning changes. In addition, for this subject group with moderate atherosclerosis and no carotid stenosis, it was assumed that no physiological changes would have occurred within the 2 week scanning session period that would change carotid measurements or increase the variability of the measurements.

Multiple MR sequences were acquired and the total acquisition time for all sequences was approximately $60 \mathrm{~min}$. At both $3.0 \mathrm{~T}$ and $1.5 \mathrm{~T}$, a set of $1002 \mathrm{~mm}$ thick axial two-dimensional (2D) time-of-flight slices was acquired in 4 min to identify the superior-inferior level of the flow dividers on each side. A localized shim was performed prior to each of the remaining 
sequences to ensure good performance of fat saturation pulses. The remaining series consisted of a multi-slice 2D black blood acquisition, with T1-weighted (T1w) contrast, using double inversion recovery (DIR with $180^{\circ}$ pulse). Parameters for the T1w scans analyzed in this study and acquired at $1.5 \mathrm{~T}$ and $3.0 \mathrm{~T}$ are provided in table 1 . All images following the localizer were acquired with $0.5 \mathrm{~mm}$ in-plane resolution, $2 \mathrm{~mm}$ slice thickness and fat saturation. The subject motion was monitored by examining odd-to-even slice mis-registration. All post-localizer series were planned with slices centered in the superior-inferior direction at a point $5 \mathrm{~mm}$ superior to the flow divider of the index bifurcation. The $\mathrm{T} 1 \mathrm{w}$ sequences were acquired with $16 \times 2 \mathrm{~mm}^{2}$ thick slices resulting in 16 contiguous slices and covering $32 \mathrm{~mm}$ in the head-foot axis.

\subsection{Image analysis and measurements}

2.3.1. MRI-derived outer wall volume, lumen volume and vessel wall volume. Carotid images acquired using the T1w sequence (both right and left carotid sides) at scan and rescan were acquired at both $1.5 \mathrm{~T}$ and $3.0 \mathrm{~T}$ field strengths and were evaluated by a single observer who was blinded to field strength, subject identity and timepoint. As field-strength-dependent differences and variability and not inter-observer variability were the focus of this study, we limited the analysis to a single observer who performed multiple measurements of all images at both field strengths. In this study, T1w scans were chosen for analysis: (1) because of previously published analysis that showed that blood flow suppression was best achieved using the single slice DIR technique (Yarnykh et al 2006) and (2) to allow for a direct comparison with previously published results (Yarnykh et al 2006). In addition, because this study focused on interscan and intra-observer variability, the single observer evaluated only T1w images in order to minimize observer memory bias that may have resulted from the analysis of multiple datasets. All carotid side images for each timepoint and field strength were reviewed on five occasions by a single observer who was blinded to subject identity, timepoint and field strength. For each measurement trial, images were analyzed in an independently randomized order and randomization was performed by a technologist and not the observer. Unlike most studies that have evaluated carotid atherosclerosis using MR imaging (Yuan et al 2006), scans acquired at the two timepoints were not reviewed together but were randomized within the entire set of images in order to provide the most conservative estimate of intra-observer and interscan variability.

Manual planimetry was used in the analysis of MR images and has been previously used to determine 3DUS VWV (Egger et al 2007) and 3DUS TPV (Landry et al 2004, Landry and Fenster 2002), both in vitro and in vivo (Egger et al 2007, Landry et al 2004, Landry and Fenster 2002). MRI-derived measurements from T1w images were determined using 3D Quantify software developed in our laboratory as previously described for 3DUS images (Ainsworth et al 2005, Landry et al 2004). This visualization software has been validated for the measurement of 3DUS carotid images and multiple observers have been trained to use the software to segment MR carotid images. The training of observers for analysis involved first $3 \mathrm{~h}$ of training with the visualization software, followed by $10 \mathrm{~h}$ of training for the method of manual segmentation and then finally, validation of variability. The validation of measurement variability involved the observer performing manual segmentation of a dataset of five carotid side images on five separate occasions with calculation of COV and ICC. Once COV and ICC calculated for the dataset were the same as an expert radiologist observer $(\mathrm{COV}<10 \%$, ICC $>96 \%$ ), the observer was considered a trained expert. Typically, observers are trained during a 6 week period including the variability analysis. For the measurements made in this study, eight subject images or 16 carotid sides were measured five times each from the T1w images. For 


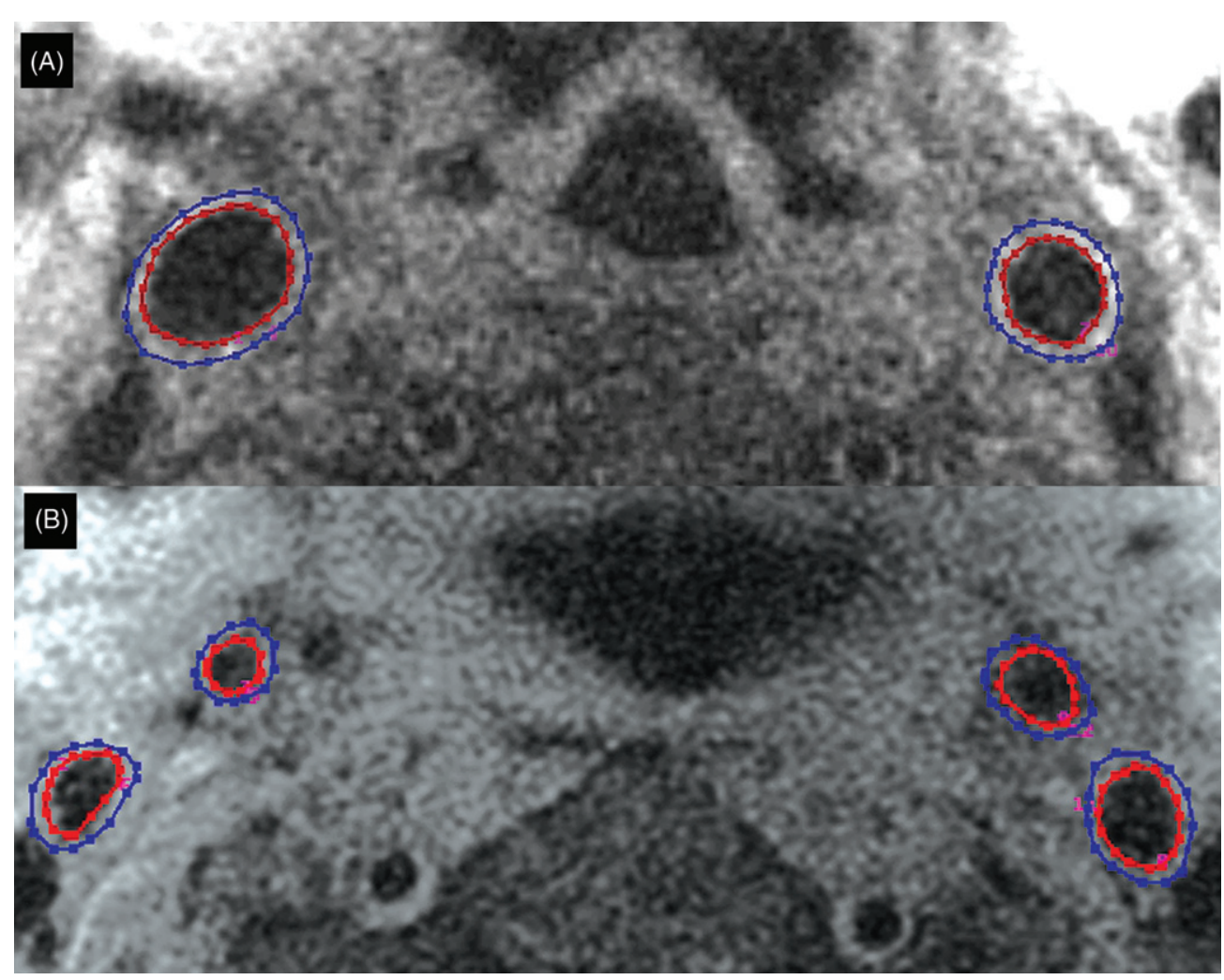

Figure 1. Carotid artery vessel wall and lumen manual segmentation. (A) Common carotid artery (CCA), (B) internal carotid artery (ICA) and external carotid artery (ECA).

all carotid images, the single observer manually outlined the lumen-vessel wall boundary (shown in red in figure 1) and the outer vessel wall-tissue boundary (shown in blue in figure 1) from $11 \mathrm{MR}$ slices segmented in $2 \mathrm{~mm}$ increments. The visualization software calculated the incremental volume separately for the lumen boundary and the outer vessel wall of the common carotid artery (figure 1(A)) and both internal and external carotid arteries (figure 1(B)). As previously described for 3DUS images (Egger et al 2007), MRI-derived VWV was calculated as the difference between the outer vessel wall volume and lumen volume.

2.3.2. Signal-to-noise ratio and contrast-to-noise ratio calculations. The signal-to-noise ratio (SNR) of the carotid artery wall and the contrast-to-noise ratio (CNR) for the tissuearterial wall boundary were calculated for both scan and rescan images acquired at $1.5 \mathrm{~T}$ and 3.0 T. As shown in figure 2, for SNR, the magnitude of the mean signal intensity $(S)$ for a single cubic region of interest located in the vessel wall of the left and right common carotid artery for each subject was calculated. For the purposes of performing all signal intensity measurements, Image J 1.37v (downloaded from http://rsb.info.nih.gov/ij/docs/index.html) was used. The standard deviation (SD) of the background noise was measured in a single region of interest (ROI) within the image where there was no signal or image artifact. For this study, the background noise for tracheal air was used. The SNR was calculated by dividing the mean signal intensity in the ROI by the standard deviation of the background 


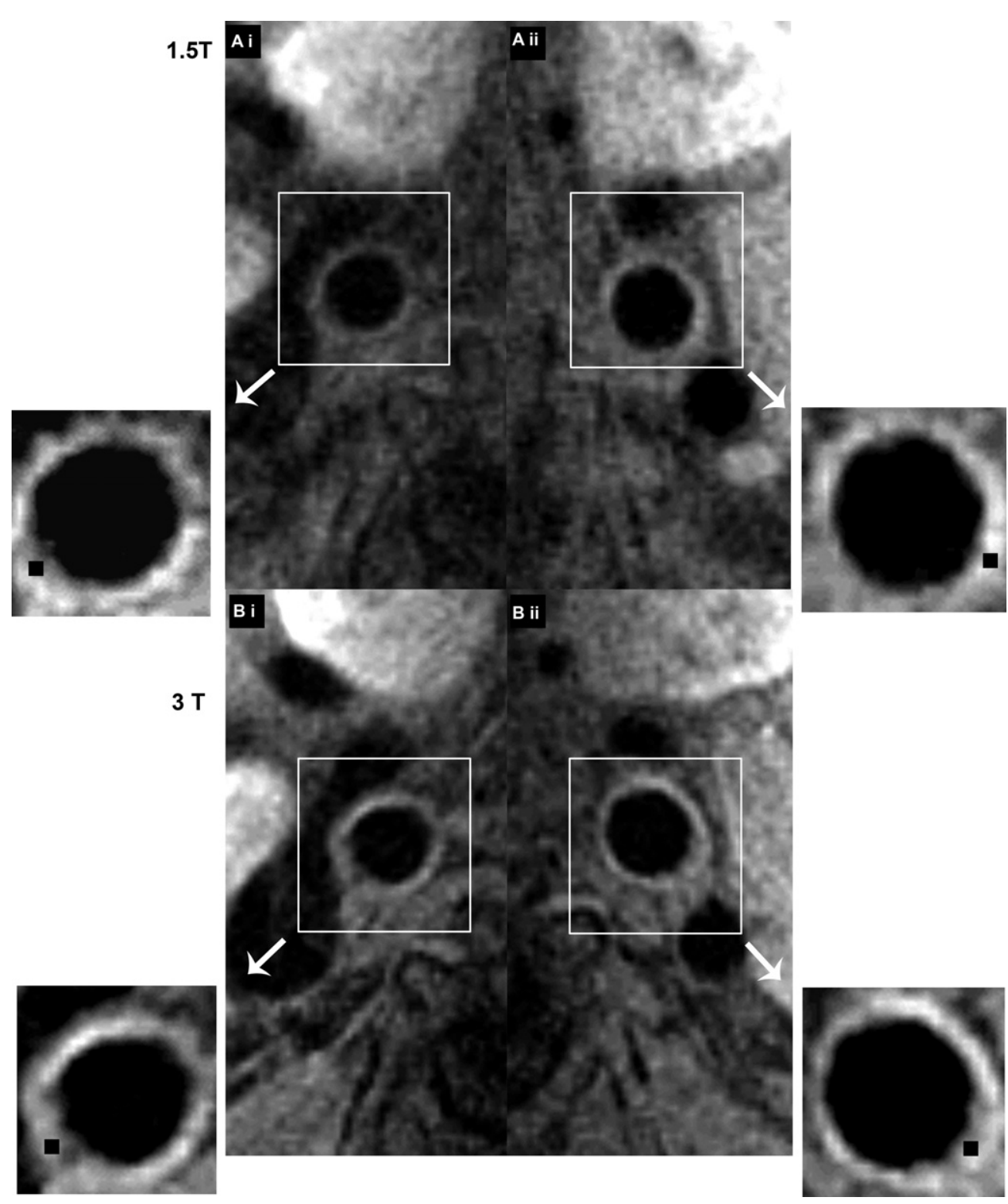

Figure 2. Representative magnetic resonance imaging of carotid artery at $1.5 \mathrm{~T}$ and $3.0 \mathrm{~T}$. (A) Common carotid artery at $15 \mathrm{~T}$ and (B) $3.0 \mathrm{~T}$, showing for both (i) right and (ii) left arteries. Arrows point to inset box magnifications of common carotid artery wall with cubic region of interest (ROI) from which signal was estimated.

noise signal intensity. CNR for the tissue-arterial wall boundary was also calculated as the difference in signal intensity in a ROI of the arterial wall and ROI of adjacent muscle tissue divided by the standard deviation of the background noise signal intensity. The SNR and CNR were calculated for all carotid images, for all subjects, at scan and rescan at both field strengths. 
Table 2. Baseline subject demographics: continuous variables are expressed as a mean \pm standard deviation. VWV is expressed as a mean of all carotid sides measured ( 16 for 8 subjects) \pm standard deviation (SD). IMT is intima-media thickness and is expressed as mean of right and left sides for each subject.

\begin{tabular}{ll}
\hline Study subjects & All evaluable subjects $n=8$ \\
\hline Discrete variables & \\
$\quad$ Male sex & 4 \\
$\quad$ Diabetes $(n)$ & 0 \\
$\quad$ Treated hypertension $(n)$ & 5 \\
$\quad$ Treated hyperlipidemia $(n)$ & 8 \\
Continuous variables & \\
Age \pm SD $(Y)$ & $66 \pm 8$ \\
$\quad$ Weight \pm SD $(\mathrm{kg})$ & $79 \pm 15$ \\
IMT \pm SD $(\mathrm{mm})$ & $0.90 \pm 0.12$ \\
MRI VWV 3.0 T \pm SD $\left(\mathrm{mm}^{3}\right)$ & $1050 \pm 230$ \\
MRI VWV 1.5 T \pm SD $\left(\mathrm{mm}^{3}\right)$ & $1190 \pm 290$ \\
\hline
\end{tabular}

\subsection{Statistical methods}

Mean carotid measures were calculated for the eight subjects (16 carotid sides) evaluated at both field strengths at scan and rescan. A two-tailed paired Student's $t$-test was performed to interrogate any significant differences between mean measurements at scan and rescan between field strengths and between the mean of scan and rescan between field strengths. A two-way completely repeated analysis of variance (ANOVA) was also used to determine mean volume differences between scan and rescan and between field strengths for outer wall volume, lumen volume and VWW. Possible interactions between timepoint and field strength were also determined.

Three different standard deviation (SD) measurements were calculated. The population standard deviation (pSD) of the mean carotid measures was calculated for the group of eight evaluated subjects (16 carotid sides) as shown in table 2. The repeated analysis standard deviation (rSD) or intra-observer standard deviation for all carotid side measurements (shown in table 2) was calculated based on the five repeated image analyses for each carotid side. The five repeated measurements for each individual carotid side were used to calculate the individual carotid side standard deviation, $\mathrm{SD}_{\mathrm{i}}$. The interscan $\mathrm{SD}$ (iSD) was calculated as the SD of the differences between scan and rescan measurements for all carotid sides (Egger et al 2007). Intra-observer COV for scan and rescan measurements were calculated using the corresponding repeated analysis standard deviation (rSD) divided by the mean of all measurements. The interscan SD (iSD) was divided by the corresponding mean value of scan and rescan to provide interscan $\mathrm{COV}$.

GraphPad Prism version 4.01 for Windows (GraphPad Software Inc., 2004) was used to perform linear regressions and Bland Altman analyses (Bland and Altman 1986). SPSS version 14.0 (SPSS Inc., 2005) was used to determine intra-class correlation coefficients (ICC) for repeated measurements of MRI VWV. Standard deviations and COV for mean scores of scan and rescan were compared for each field strength using the Wilcoxon ranks signed test, a method used to analyze non-normal data, which is often the case with scores derived from ratios. Simple regressions were compared using the Fisher's $Z$ transformation test (FZTT). The square root of $R^{2}$ was first transformed before performing the FZTT. The mean SNR and mean CNR values (of 16 carotid sides) were compared using the Student's $t$-test and the Wilcoxon ranks signed test. The statistical outcome for all tests was considered significant when the probability of making a type I error was less than $5 \%(p<0.05)$. 
Table 3. Common carotid vessel wall signal-to-noise ratio (SNR) and contrast-to-noise ratio for outer vessel wall-tissue boundary $\left(\mathrm{CNR}_{\mathrm{wt}}\right)$ at scan and rescan $3.0 \mathrm{~T}$ and $1.5 \mathrm{~T}$.

\begin{tabular}{|c|c|c|c|c|c|c|c|c|}
\hline \multirow[b]{2}{*}{ Parameter } & \multicolumn{2}{|c|}{$1.5 \mathrm{~T}$} & \multicolumn{2}{|c|}{$3.0 \mathrm{~T}$} & \multicolumn{2}{|c|}{$\begin{array}{c}\text { Significant } \\
\text { difference } \\
1.5 \mathrm{~T}-3.0 \mathrm{~T}(p)\end{array}$} & \multicolumn{2}{|c|}{$3.0 \mathrm{~T} / 1.5 \mathrm{~T}$} \\
\hline & Scan & Rescan & Scan & Rescan & Scan & Rescan & Scan & Rescan \\
\hline $\mathrm{SNR}( \pm \mathrm{SD})$ & $17(9)$ & $18(6)$ & 32 (19) & $32(16)$ & 0.001 & 0.002 & $1.9(0.8)$ & $1.8(0.7)$ \\
\hline $\mathrm{CNR}_{\mathrm{wt}}( \pm \mathrm{SD})$ & $12(6)$ & $9(6)$ & $15(5)$ & $11(6)$ & 0.01 & 0.05 & $1.7(0.8)$ & $1.6(0.9)$ \\
\hline
\end{tabular}

\section{Results}

\subsection{Study subjects}

All ten subjects who were enrolled in the study completed all imaging sessions at both $1.5 \mathrm{~T}$ and 3.0 T. All ten subject images at scan and rescan acquired at $3.0 \mathrm{~T}$ were evaluable, but only eight of ten subject images acquired at $1.5 \mathrm{~T}$ were evaluable due to motion artifacts in images for two subjects at the scan timepoint. Thus, we analyzed images for eight subjects (16 carotid sides) who had evaluable scan and rescan images acquired at both $1.5 \mathrm{~T}$ and 3.0 T. Baseline demographic characteristics (reported at baseline scan) are provided in table 2 for the eight subjects ( 16 carotid sides) evaluated in this study. There were no reported clinical events for any subject between imaging sessions.

\subsection{Signal-to-noise ratio and contrast-to-noise ratio calculations}

Figure 2 provides a graphical representation of how the SNR was calculated for this study, while direct visual comparison of the SNR for two representative subjects is provided in figure 3 for the common carotid artery (CCA) and for the internal carotid artery (ICA) and external carotid artery (ECA) at scan and rescan for both field strengths. Table 3 shows the mean SNR and CNR values for all carotid side images evaluated at both $1.5 \mathrm{~T}$ and $3.0 \mathrm{~T}$. The mean increase in the SNR and CNR at 3.0 T as compared to $1.5 \mathrm{~T}$ and the significance of the difference between $1.5 \mathrm{~T}$ and 3.0 T results is also shown. As shown in table 3, the mean increase in the SNR for images acquired at $3.0 \mathrm{~T}$ as compared to $1.5 \mathrm{~T}$ was $90 \%$ at scan $(1.9 \pm$ $0.8(p<0.001))$ and $80 \%$ at rescan $(1.8 \pm 0.7(p<0.002))$. The increase in outer wall-tissue CNR for images acquired at $3.0 \mathrm{~T}$ as compared to $1.5 \mathrm{~T}$ was $70 \%$ at scan $(1.7 \pm 0.8(p<$ $0.01))$ and $60 \%$ at rescan $(1.6 \pm 0.9(p<0.05))$.

\subsection{Measurements of the outer wall volume, lumen volume and vessel wall volume}

Direct comparison of the absolute value and standard deviations of all scan and rescan measurements, the interscan differences, as well as the corresponding COV and ICC values for repeated measures are provided in table 4. The difference between $1.5 \mathrm{~T}$ and $3.0 \mathrm{~T}$ measures for mean outer wall was $54 \pm 138 \mathrm{~mm}^{3}$, for mean lumen was $54 \pm 98 \mathrm{~mm}^{3}$ and for mean VWV was $108 \pm 105 \mathrm{~mm}^{3}$. A two-tailed paired Student's $t$-test showed a significant difference between the VWV measurements made at $1.5 \mathrm{~T}$ and $3.0 \mathrm{~T}$. Separate two-way completely repeated ANOVAs for timepoint (scan or rescan) and field strength was performed for: (1) outer wall volume, which is the entire volume encompassed by the outer wall-tissue segmented boundary, (2) lumen volume, which is the entire volume encompassed by the lumen-wall segmented boundary and (3) vessel wall volume (VWV), the value of the lumen 


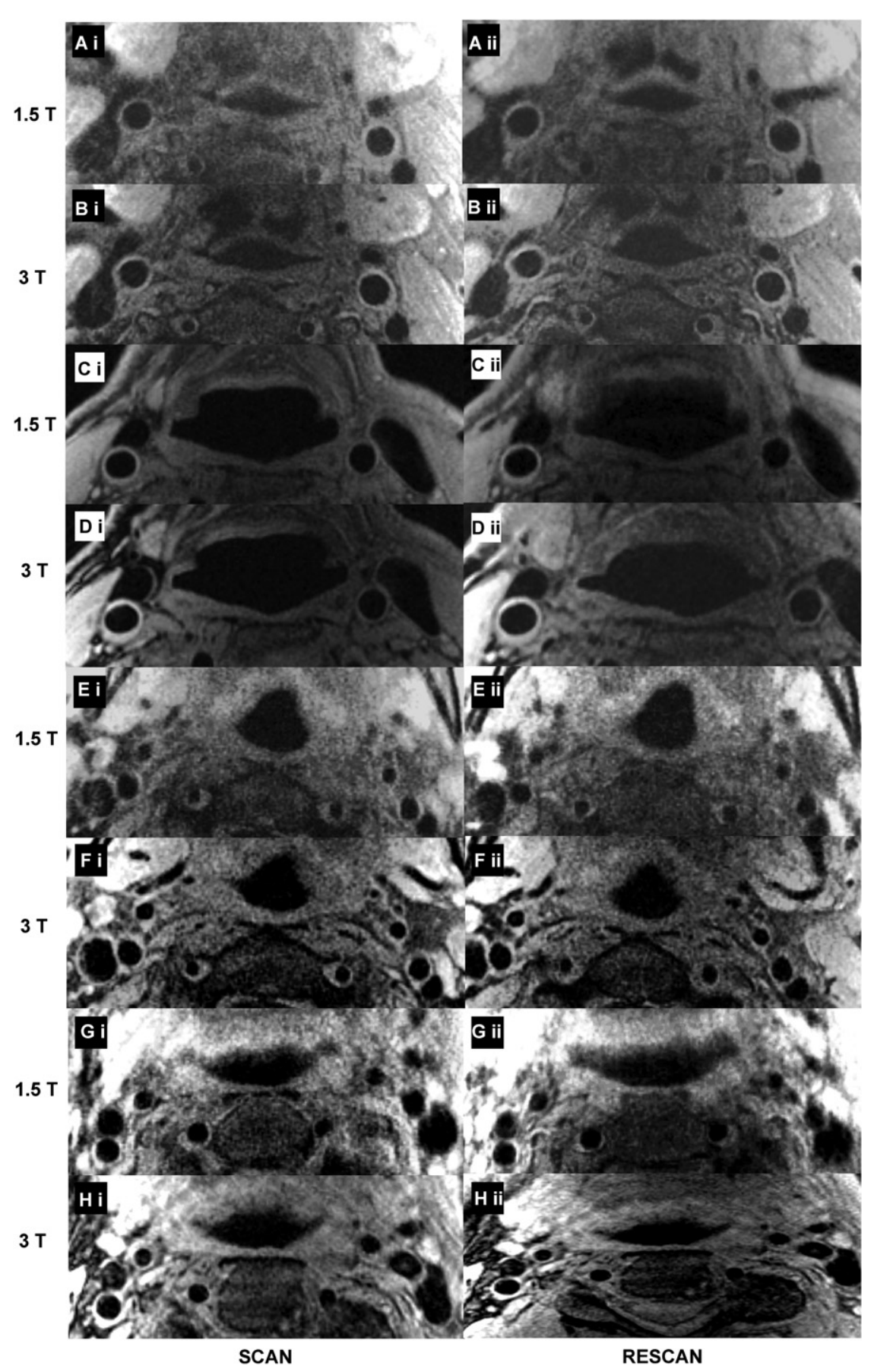

Figure 3. Scan and rescan carotid artery magnetic resonance imaging scans acquired $1.5 \mathrm{~T}$ and 3.0 T. (A and C) Common carotid $1.5 \mathrm{~T}$, (i) scan, (ii) rescan for two representative subjects. (B and D) common carotid $3.0 \mathrm{~T}$, (i) scan, (ii) rescan for two representative subjects. (E and G) Internal and external carotid 1.5 T, (i) scan, (ii) rescan for subjects shown in (A-D). (F and H) Internal and external carotid 3.0 T, (i) scan, (ii) rescan for subjects shown in (A-D). 

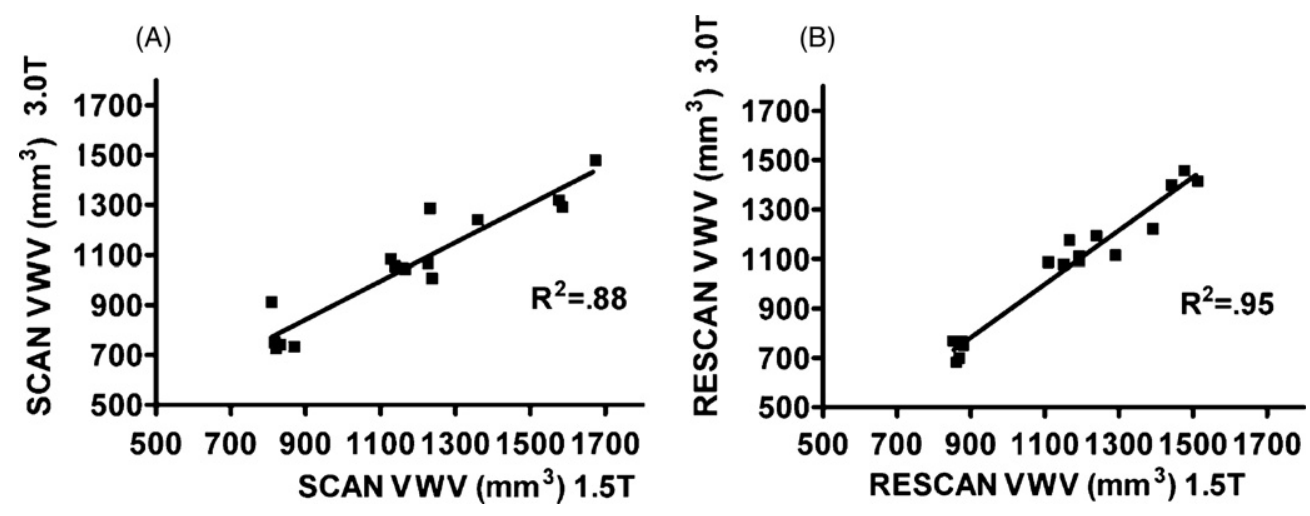

Figure 4. Linear regression of measurement of VWV at $1.5 \mathrm{~T}$ and $3.0 \mathrm{~T}$ for scan and rescan: (A) scan, $\left(y=0.77 x+1.50\right.$ and $R^{2}=0.88$; (B) rescan, $\left.y=1.08 x-191\right)$ and $R^{2}=0.95$. Difference between $R^{2}$ at scan and rescan not significant.

volume subtracted from the outer wall volume. A significant main effect for field strength was found for all measurements (outer wall volume $p<0.05$, lumen volume $p<0.01$, VWV $p<0.001$ ). There was no effect found for timepoint (scan or rescan) at either field strength. Linear regression was also performed for the $16 \mathrm{VWV}$ measurements acquired at $1.5 \mathrm{~T}$ and $3.0 \mathrm{~T}$ and the results (figure 4) show $R^{2}$ values of 0.88 for scan VWV and 0.95 for rescan. These regression $\left(R^{2}\right)$ values were not significantly different as determined using the Fisher's $Z$ test.

\subsection{Intra-observer variability}

Intra-observer variability was assessed based on the blinded and randomized repeated measurements using COV and ICC (table 4). The standard deviations (SD), COV and ICC values showed no significant difference between field strengths.

\subsection{Interscan variability}

Interscan variability for the lumen volume, outer wall volume as well as VWV was assessed using interscan (IS) COV and ICC also shown in table 4. Interscan COV for VWV at $1.5 \mathrm{~T}$ was $5.7 \%$ whereas at $3.0 \mathrm{~T}$ it was $7.8 \%$ and this difference was not significant. Linear regression (figure 5) showed a strong correlation between scan and rescan VWV measurements for $1.5 \mathrm{~T}\left(R^{2}=0.96\right)$ and $3.0 \mathrm{~T}\left(R^{2}=0.87\right)$. The scan-rescan regression values at $1.5 \mathrm{~T}$ and $3.0 \mathrm{~T}$ were not significantly different as determined using the Fisher's $Z$ test. Bland Altman plots (figure 6) for interscan differences at $1.5 \mathrm{~T}$ and $3.0 \mathrm{~T}$ provide visual evidence of similar scan-rescan measurement variability for all 16 carotid images at both field strengths.

\section{Discussion}

\subsection{Signal-to-noise and contrast-to-noise at $1.5 \mathrm{~T}$ and $3.0 \mathrm{~T}$}

Analysis of the T1w images acquired in this study showed that the SNR values at $3.0 \mathrm{~T}$ were between $80 \%$ (rescan) to $90 \%$ increased (scan) as compared to images acquired at $1.5 \mathrm{~T}$, which is in good agreement with the theoretically predicted two-fold difference (Hoult and Lauterbur 1979), and previously published results (Koktzoglou et al 2006, Yarnykh et al 2006). For T1w imaging such as analyzed in this study, the SNR gains are expected to be less than two 
(A)

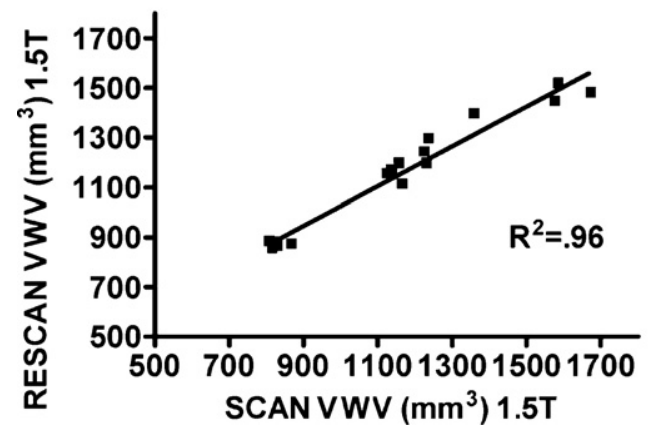

(B)

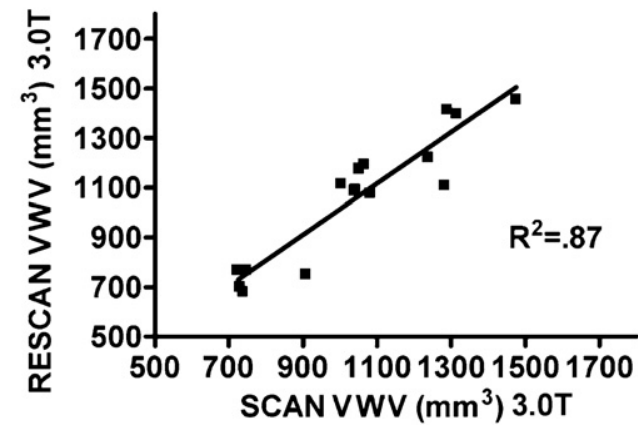

Figure 5. Scan-rescan linear regressions of VWV measurement at $1.5 \mathrm{~T}$ and $3.0 \mathrm{~T}$. (A) $1.5 \mathrm{~T}, y=$ $0.80 x+226$ and $R^{2}=0.96$, (B) $3.0 \mathrm{~T}, y=1.03 x-20$ and $R^{2}=0.88$. Difference between $R^{2}$ at scan and rescan not significant.

Table 4. Repeated measures of carotid outer wall volume, lumen volume and vessel wall volume $($ IS $=$ interscan).

\begin{tabular}{|c|c|c|c|c|c|c|}
\hline & \multicolumn{3}{|c|}{$1.5 \mathrm{~T}$} & \multicolumn{3}{|c|}{$3.0 \mathrm{~T}$} \\
\hline & Scan & Rescan & IS & Scan & Rescan & IS \\
\hline \multicolumn{7}{|l|}{ Outer wall } \\
\hline Volume $\left(\mathrm{mm}^{3}\right)( \pm \mathrm{SD})$ & $2084(88)$ & $2059(74)$ & $2071(95)$ & $2030(69)$ & 2003 (67) & $2017(100)$ \\
\hline $\mathrm{COV} \%$ & 4.5 & 3.6 & 4.6 & 3.4 & 3.3 & 5.0 \\
\hline ICC & 0.97 & 0.97 & 0.98 & 0.98 & 0.98 & 0.96 \\
\hline \multicolumn{7}{|l|}{ Lumen } \\
\hline Volume $\left(\mathrm{mm}^{3}\right)( \pm \mathrm{SD})$ & $918(36)$ & $901(31)$ & $909(53)$ & $984(26)$ & $942(25)$ & $963(83)$ \\
\hline COV\% & 3.9 & 3.4 & 5.8 & 2.6 & 2.7 & 8.6 \\
\hline ICC & 0.98 & 0.98 & 0.98 & 0.99 & 0.99 & 0.95 \\
\hline \multicolumn{7}{|l|}{ VWV } \\
\hline Volume $\left(\mathrm{mm}^{3}\right)( \pm \mathrm{SD})$ & $1165(91)$ & $1157(79)$ & $1161(66)$ & $1046(81)$ & $1061(68)$ & $1053(82)$ \\
\hline $\mathrm{COV} \%$ & 7.8 & 6.8 & 5.7 & 7.7 & 6.4 & 7.8 \\
\hline ICC & 0.88 & 0.87 & 0.96 & 0.88 & 0.92 & 0.94 \\
\hline
\end{tabular}

due to the fact that $\mathrm{T} 1$ values for most tissues are longer at $3.0 \mathrm{~T}$ than at $1.5 \mathrm{~T}$ and because for this study the same relaxation time (TR) was used at both field strengths. We found that CNR values at $3.0 \mathrm{~T}$ were also $60 \%$ (scan) to $70 \%$ (rescan) greater than images acquired at $1.5 \mathrm{~T}$. Taken together, these results provide a foundation for comparison of intra-observer measurement and interscan measurement variability in this study.

\subsection{Carotid measurements}

As previously described (Yarnykh et al 2006) we detected no significant difference in mean volume values at scan and rescan. However, for both scan and rescan measurements, the mean at both field strengths was significantly different as assessed using a paired two-tailed Students $t$-test. Additionally, separate two-way repeated ANOVAs of all measurements acquired at scan and rescan and at each field strength showed a significant main effect for field strength on all volume measurements assessed. This result was not anticipated, especially in the light of previous work by Yuan and co-workers (Yarnykh et al 2006) that showed no significant difference between morphological values measured at $1.5 \mathrm{~T}$ and 3.0 T. Although the differences 


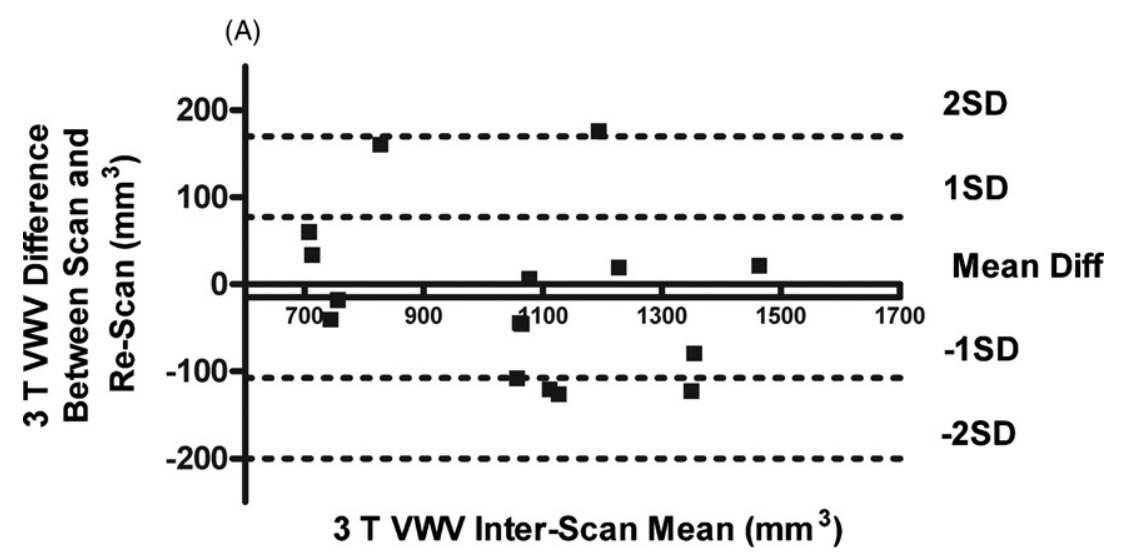

(B)

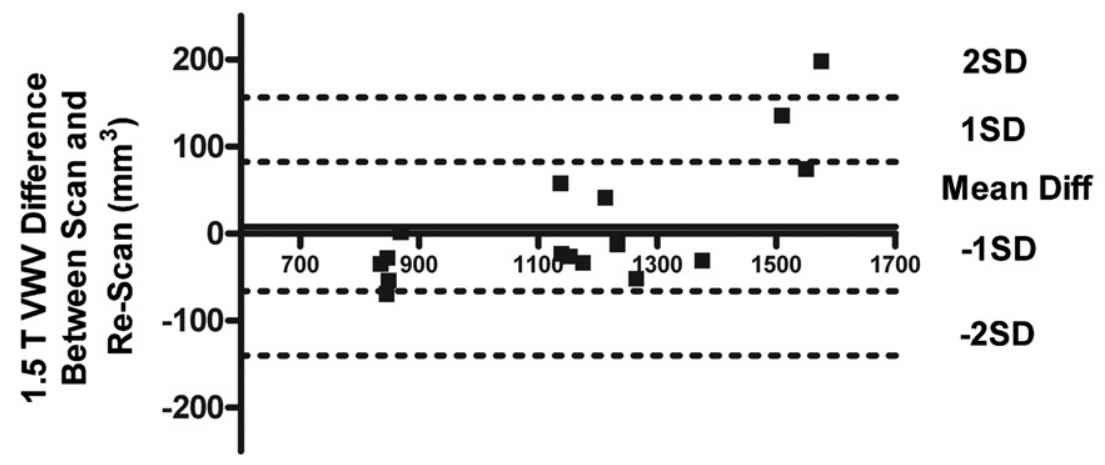

1.5 T VWV Inter-Scan Mean $\left(\mathrm{mm}^{3}\right)$

Figure 6. Bland Altman plots of VWV difference between scan and rescan VWV measurements for the eight subjects (2 sides per subject) at scan and rescan at: (A) $1.5 \mathrm{~T}$, (B) $3.0 \mathrm{~T}$.

in the measurements themselves were not the focus of this study, repeated measures analysis showed that the lumen volumes at 3.0 T were measured as larger than at $1.5 \mathrm{~T}$. Hence overall VWV was lower at 3.0 T as measured than at 1.5 T. Possible reasons for the difference include the fact that in our study we employed repeated observer measurements of scan and rescan images, which is statistically more powerful and increases the probability of significantly detecting differences in the volumes examined. Moreover, repeated measurements allow ANOVA to detect significant differences that would not be detected using independent subject tests. In addition, we analyzed all slices in the carotid, including the common, internal and external carotid artery. This has not been directly assessed in previously published studies and it is possible that the differences detected in volumes between field strengths may reflect observer interpretation of the enhanced lumen-wall and adventitia-tissue boundaries which may have biased the interpretation of the $1.5 \mathrm{~T}$ and $3.0 \mathrm{~T}$ images. It further suggests that care must be taken in order to maintain analysis of images from datasets acquired from the same field strength.

\subsection{Lumen volume, outer wall volume and VWV variability}

In this study, we assessed both intra-observer and interscan variability based on the blinded and randomized five repeated measurements of all subject carotid image sides at both field 
strengths $(8$ subjects $\times 2 \mathrm{CS} \times 2$ field strengths $\times 2$ timepoints $\times 5$ repeated measures $=$ 320 measurements), for the common, internal and external carotid artery segments. The standard deviations (SD) of the single observer repeated measurements as well as COV were compared and showed no significant differences between scans or field strengths. This suggests that the SNR and CNR at both field strengths were sufficient for the observer to identify and trace the vessel and lumen boundaries precisely and therefore that neither SNR nor CNR is the dominant factor in the variability of MR measurements like VWV extracted from T1w images. These results suggest that despite the significant improvement in SNR, overall interscan and intra-observer variability was not improved for images acquired at $3.0 \mathrm{~T}$ as compared to those acquired at 1.5 T. Linear regressions of scan and rescan VWV measurements shown in figure 5 for $1.5 \mathrm{~T}\left(R^{2}=0.96\right)$ and $3.0 \mathrm{~T}\left(R^{2}=0.87\right)$ were also not significantly different. While this can be interpreted as suggesting no significant improvement in interscan measurement variability at 3.0 T, there are limitations in the interpretation of $\mathrm{R} 2$ values. Hence the finding of no significant field strength by timepoint interaction shown by separate two-way ANOVA for volumes measured at scan, rescan and across the two field strengths provides further evidence that field strength did not play a role in the scan-rescan volume measurement variability.

In this study we scanned and assessed ten subjects with moderate atherosclerosis in order to assess variability in subjects most likely to be encountered in clinical practice and clinical research studies of carotid atherosclerosis. Although ten subjects completed the study, eight subjects were evaluable at both field strengths and timepoints because of motion artifacts in the images of two subjects, providing a total of 16 vessels to analyze. The requirement in MR imaging for subject compliance with respect to motion is critical and our result is typical of other studies at our center and other centers where images, image slices and subjects must be removed from the analysis population because of image quality issues. While the sample size for the current study was not large $(n=8$ subjects $\times 2$ sides $\times 2$ scans $\times 2$ field strengths $=$ 64 carotid sides $\times 5$ repeated measures $=320$ measurements), the repeated measures analysis we employed detected differences in measurements between field strengths and no difference in variability. While the inclusion of more subjects or more rescan timepoints would have increased the power to detect differences, increased subject recruitment and retention, which was already very complex in the current protocol, would not have been practicable (i.e. more scanning visits at both field strengths). For this study, we focused on a complete repeated measures analysis of a single type of MR image (T1w), which we believe is in accordance with the requirement for rapid volumetric analysis in multi-center clinical research studies. Another avenue to explore in future is the analysis of other images acquired during the MR scanning sessions in order to assess whether our results with T1w black blood images are representative of the interscan variability observed for the analysis of other types of images acquired (Dehnavi et al 2007, Yarnykh et al 2006).

Why does not the significant improvement in the SNR and CNR of carotid images acquired at 3.0 T result in a lower intra-observer and interscan variability of carotid VWV? One possible explanation is that the mean SNR of $17 \pm 9$ (scan) and $16 \pm 6$ (rescan) and the mean CNR of $12 \pm 6$ (scan) and $9 \pm 6$ (rescan) at $1.5 \mathrm{~T}$ are necessary and sufficient for observers to make a judgment regarding where to manually segment the lumen-wall and outer arterial wall-tissue boundaries. In other words, this study suggests that SNR and CNR are not the limiting factors that drive measurement variability, and perhaps observer interpretation and reinterpretation is the major limiting factor in the analysis of MR images of SNR greater than 15 .

The improvement in SNR that is theoretically possible (Hoult and Lauterbur 1979), experimentally shown here and elsewhere (Cury et al 2006, Koktzoglou et al 2006, Yarnykh et al 2006)), does not provide any further aid to the observer in the task of identifying the boundary. However, because of the improved SNR, higher field strengths such as those 
provided by $3.0 \mathrm{~T}$ may provide an advantage in the segmentation and volumetric analysis of interior plaque components, as well as in the analysis of plaque surface morphology, providing a rationale for further assessment of the influence of field strength in carotid atherosclerosis imaging. It is also possible that with the advent of semi- or fully-automated boundary extraction/tracing methods, the potential benefits of SNR and CNR increase with higher field strengths may be exploited. Another potential but yet untested benefit of working at higher field strengths would be the ability to scan subjects in almost 4-times shorter timeframes, incurring nearly 2-times lower SNR, and perhaps still achieving the same measurement variability. This has important indications for future clinical trial designs and costs, since a dominant factor in imaging studies is the deletion of scans from analysis due to motion artifacts, for which scan time is the strongest predictor.

\section{Conclusions}

This study compared the intra-observer and interscan variability of carotid atherosclerosis measurements derived from images acquired at $1.5 \mathrm{~T}$ and $3.0 \mathrm{~T}$. We attempted to simulate conditions typically encountered in longitudinal studies of patients with carotid atherosclerosis in order to assess both interscan and intra-observer variability. While both SNR and CNR were significantly greater in images acquired at 3.0 T, intra-observer variability assessed using COV and ICC of mean VWV was not significantly improved. Similarly, interscan variability assessed at both field strengths assessed using COV, and linear regression was not significantly different. Taken together, these results suggest that although images acquired 1.5 T MRI having lower SNR and CNR, interscan and intra-observer variability measured was not significantly different from images acquired at 3.0 T. A two-way analysis of variance for measurement volumes at all timepoints and field strengths also showed a small but significant difference in the volumes calculated at $1.5 \mathrm{~T}$ and 3.0 T, but no difference between volumes measured at scan or rescan.

\section{Acknowledgments}

AV acknowledges stipend training support from the Western Graduate Research Fund (The University of Western Ontario) and an Ontario Graduate Scholarship in Science \& Technology (AV) as well as a fellowship from the Canadian Institutes of Health Research Strategic Training Program in Vascular Research. Operational funding is gratefully acknowledged from the Ontario Research and Development Challenge Fund, Pfizer, Virtual Scopics Ltd, and the Canadian Institutes of Health Research. The authors acknowledge the expert assistance of James Kost MSc, Chris Blake MSc, Shayna McKay BSc, and Rhonda Walcarius RT(MR). AF acknowledges the support of the Canada Foundation for Innovation and holds a Tier 1 Canada Research Chair in Biomedical Engineering. BKR gratefully acknowledges salary support from the Barnett-Ivey Heart and Stroke Foundation of Ontario Endowed Chair award and operational support in part from the Canadian Institutes of Health Research grant GR-14973.

\section{References}

Ainsworth C D, Blake C C, Tamayo A, Beletsky V, Fenster A and Spence J D 2005 3D ultrasound measurement of change in carotid plaque volume: a tool for rapid evaluation of new therapies Stroke 36 1904-9

Arntzenius A C, Kromhout D, Barth J D, Reiber J H, Bruschke A V, Buis B, van Gent C M, Kempen-Voogd N, Strikwerda S and van der Velde E A 1985 Diet, lipoproteins, and the progression of coronary atherosclerosis: the Leiden intervention trial N. Engl. J. Med. 312 805-11 
Ashrafian H, Lim T K and Senior R 2007 Carotid ultrasound imaging: an effective technique for detecting early atherosclerosis-vascular screening for cardiovascular disease has come of age J. Am. Soc. Echocardiogr. 20 1-3

Barnett H J et al 1998 Benefit of carotid endarterectomy in patients with symptomatic moderate or severe stenosis: North American symptomatic carotid endarterectomy trial collaborators N. Engl. J. Med. 339 1415-25

Barth J D 2004 Carotid intima media thickness and beyond Curr. Drug Targets Cardiovasc. Haematol. Disord. 4 129-45

Bland J M and Altman D G 1986 Statistical methods for assessing agreement between two methods of clinical measurement Lancet 1 307-10

Blankenhorn D H and Hodis H N 1994 George Lyman Duff Memorial Lecture arterial imaging and atherosclerosis reversal Arterioscler. Thromb. 14 177-92

Blankenhorn D H, Nessim S A, Johnson R L, Sanmarco M E, Azen S P and Cashin-Hemphill L 1987 Beneficial effects of combined colestipol-niacin therapy on coronary atherosclerosis and coronary venous bypass grafts JAMA $2573233-40$

Brensike J F, Levy R I, Kelsey S F, Passamani E R, Richardson J M, Loh I K, Stone N J, Aldrich R F, Battaglini J W and Moriarty D J 1984 Effects of therapy with cholestyramine on progression of coronary arteriosclerosis: results of the NHLBI type II coronary intervention study Circulation 69 313-24

Cury R C, Houser S L, Furie K L, Stone J R, Ogilvy C S, Sherwood J B, Muller J E, Brady T J and Hinton D P 2006 Vulnerable plaque detection by 3.0 tesla magnetic resonance imaging Invest. Radiol. 41 112-5

Dehnavi R A, Doornbos J, Tamsma J T, Stuber M, Putter H, van der Geest R J, Lamb H J and de Roos A 2007 Assessment of the carotid artery by MRI at $3 \mathrm{~T}$ : a study on reproducibility J. Magn. Reson. Imaging $251035-43$

Egger M, Spence J D, Fenster A and Parraga G 2007 Validation of 3D ultrasound vessel wall volume: an imaging phenotype of carotid atherosclerosis Ultrasound Med. Biol. 33 905-14

Hatsukami T S, Thackray B D, Primozich J F, Ferguson M S, Burns D H, Beach K W, Detmer P R, Alpers C, Gordon D and Strandness D E Jr 1994 Echolucent regions in carotid plaque: preliminary analysis comparing three-dimensional histologic reconstructions to sonographic findings Ultrasound Med. Biol. 20 743-9

Hoult D I and Lauterbur P C 1979 Sensitivity of the zeugmatographic experiment involving human samples J. Magn. Reson. Imaging 34 425-33

Koktzoglou I, Chung Y C, Mani V, Carroll T J, Morasch M D, Mizsei G, Simonetti O P, Fayad Z A and Li D 2006 Multislice dark-blood carotid artery wall imaging: a $1.5 \mathrm{~T}$ and $3.0 \mathrm{~T}$ comparison J. Magn. Reson. Imaging 23 699-705

Landry A and Fenster A 2002 Theoretical and experimental quantification of carotid plaque volume measurements made by three-dimensional ultrasound using test phantoms Med. Phys. 29 2319-27

Landry A, Spence J D and Fenster A 2004 Measurement of carotid plaque volume by 3-dimensional ultrasound Stroke 35 864-9

Landry A, Spence J D and Fenster A 2005 Quantification of carotid plaque volume measurements using 3D ultrasound imaging Ultrasound Med. Biol. 31 751-62

Steinke W and Hennerici M 1989 Three-dimensional ultrasound imaging of carotid artery plaques J. Cardiovasc. Technol. 8 15-22

Yarnykh V L, Terashima M, Hayes C E, Shimakawa A, Takaya N, Nguyen P K, Brittain J H, McConnell M V and Yuan C 2006 Multicontrast black-blood MRI of carotid arteries: comparison between 1.5 and 3 tesla magnetic field strengths J. Magn. Reson. Imag. 23 691-8

Yuan C et al 2006 MRI of atherosclerosis in clinical trials NMR Biomed. 19 636-54 